INPLASY

PROTOCOL

To cite: Han et al. Efficacy and safety of Hanchuan Zupa Granule in treating asthma: A protocol for systematic review and meta-analysis. Inplasy protocol 202160052. doi: 10.37766/inplasy2021.6.0052

Received: 16 June 2021

Published: 16 June 2021

Corresponding author: Jing Han

853579561@qq.com

Author Affiliation:

Beijing University of Chinese Medicine.

Support: None.

Review Stage at time of this submission: Preliminary searches.

Conflicts of interest: None declared.

\section{Efficacy and safety of Hanchuan Zupa Granule in treating asthma: A protocol for systematic review and meta-analysis}

Han, J1; Jia, Y2; Lin, J3.

Review question / Objective: Purpose of meta-analysis of RCTs is to evaluate the clinical efficacy and safety systematically of Hanchuan Zupa granule in the treatment of bronchial asthma.

Condition being studied: Asthma is a serious global health problem affecting all age groups in human. There are at least $\mathbf{3 0 0}$ million asthmatic patients in the world, including about $\mathbf{3 0}$ million in China. At present, routine therapy for asthma is Inhaled corticosteroids (ICS) with long-acting beta-2 agonist (LABA).However, systemic side-effects that may be seen with long-term, high-dose ICS include an increase beyond the usual age-related risk of osteoporosis, cataracts and glaucoma; and adrenal suppression. In recent years, studies have found that Hanchuan Zupa Granule has potential clinical efficacy in the treatment of asthma. However, no specific recommendation of efficacy and safety has been made for asthma treatment with Hanchuan Zupa Granule in China.

INPLASY registration number: This protocol was registered with the International Platform of Registered Systematic Review and Meta-Analysis Protocols (INPLASY) on 16 June 2021 and was last updated on 16 June 2021 (registration number INPLASY202160052).

\section{INTRODUCTION}

Review question / Objective: Purpose of meta-analysis of RCTs is to evaluate the clinical efficacy and safety systematically of Hanchuan Zupa granule in the treatment of bronchial asthma.
Condition being studied: Asthma is a serious global health problem affecting all age groups in human. There are at least 300 million asthmatic patients in the world, including about 30 million in China. At present, routine therapy for asthma is Inhaled corticosteroids (ICS) with long- 
acting beta-2 agonist (LABA).However, systemic side-effects that may be seen with long-term, high-dose ICS include an increase beyond the usual age-related risk of osteoporosis, cataracts and glaucoma; and adrenal suppression. In recent years, studies have found that Hanchuan Zupa Granule has potential clinical efficacy in the treatment of asthma. However, no specific recommendation of efficacy and safety has been made for asthma treatment with Hanchuan Zupa Granule in China.

\section{METHODS}

Participant or population: Patients with bronchial asthma.

Intervention: Single drug application of Hanchuan Zupa granule or combined with other conventional therapies for asthma.

Comparator: Blank control, conventional therapies, or placebo combined with conventional therapies.

Study designs to be included: Randomized controlled trials (RCTs) will be included.

Eligibility criteria: 1. Types of study. We will collect all the RCTs of Hanchuan Zupa Granule in treatment of bronchial asthma, regardless of blinding, publication status, region, but Language will be restricted to Chinese and English. 2. Research object. The patients were clearly diagnosed as bronchial asthma, regardless of nationality, race, age, gender and course of disease. 3 . Interventions. The treatment group was treated with Hanchuan Zupa Granule in combination with conventional treatment, and control group was treated with conventional therapy. Conventional therapies include ICS, LABA, Short-acting beta-2 agonist(SABA) and theophylline and so on. There was no limit on the types, dosage, frequency and course of conventional therapy.

Information sources: We will search articles in seven electronic database including Cochrane Library, PubMed, EMBASE, CNKI, CBM, VIP and Wangfang. All publications until 31 May 2021 will be searched without any restriction of languages or article type.

Main outcome(s): One of main outcomes is Pulmonary function, including Forced expiratory volume in one second (FEV1), forced vital capacity(FVC), FEV1/FVC(\%), peak expiratory flow(PEF). The other main outcome is clinical efficacy rate, which is defined as the ratio of effective to total patients. Efficacy or not will be judged by the researchers in publications.

Additional outcome(s): Inflammatory factors (IL-4, IL-6, TNF-a), arterial blood gas analysis (PCO2, PO2), and the incidence of adverse events.

Quality assessment / Risk of bias analysis: Two researchers will assess the risk of bias independently of included studies. Any disagreement will be resolved by discussion until consensus is reached or by consulting a third researcher in assessment. If the results or data of articles are insufficient to meet the our requirements of pool statistics, correspondent authors will be contacted by e-mail. Two researchers will assess the risk of bias in trials according to the guidance in the Cochrane Handbook for Systematic Reviews of Interventions, based on seven it e m s: (1) random sequence generation(selection bias); (2) allocation concealment(selection bias); (3) blinding of participants and personnel (performance bias); (4) blinding of outcome assessment (detection bias); (5) incomplete outcome data(attrition bias); (6) selective reporting(reporting bias); (7) other sources of bias. Each item is classified as "Iow risk", "high risk" or "unclear", and the reasons will be recorded to support its judgment.

Strategy of data synthesis: RevMan5.3 software is used to pool data included. "Rate" or Classification Data in articles will be pooled by the Relative Risk(RR), and Quantitative Data will be pooled by the Mean Difference (MD) or Standard Mean Difference (SMD). $I^{2}$ test was used to 
evaluate the heterogeneity between studies. Less than $50 \%$ of $\mathrm{I}^{2}$ means low heterogeneity between studies and Fixed Effects Model is used to pool data, otherwise random effect model. If there is high heterogeneity in pooling, Sensitivity Analysis will be used to describe robustness of pooled results. Forest Map was used to show overall effects of Hanchuan Zupa Granule in treating asthma, and Funnel Chart was used to show the publication bias between the studys.

Subgroup analysis: None planned.

Sensitivity analysis: None planned.

Country(ies) involved: China.

Keywords: Hanchuan Zupa Granule, asthma, protocol, randomized controlled trials, systematic review, meta-analysis.

Contributions of each author:

Author 1 - Jing Han.

Author 2 - Yankun Jia.

Author 3 - Jiangtao Lin. 